\title{
Evolution of Crew Escape System configuration
}

\author{
Juluri Sreenivasulu*, Sanjoy Kumar Saha, M. M. Patil and V. Ashok \\ Aeronautics Entity, Vikram Sarabhai Space Centre, Department of Space, ISRO Post, Thiruvananthapuram 695 022, India
}

\begin{abstract}
The Crew Escape System (CES) is used for safe return of crew in case of any malfunctioning of a launch vehicle during ascent, specially in the atmospheric phase of flight. In the present case, as no active control is employed, the aerodynamic stability of CES is essential for safe return of crew in case of any emergency during ascent phase. While finalizing the shape of CES, besides stability, other aerodynamic parameters like acoustic, thermal, structural load, etc. need to be analysed. Based on detailed studies using CFD, a conical-shaped CES has been finalized. This article discusses the challenges involved in aerodynamic shaping of CES and different parameters studied while finalizing the configuration.
\end{abstract}

Keywords: Aerodynamic coefficients, Crew Escape System, launch vehicle, static margin, stability.

\section{Introduction}

THE Crew Escape System (CES) is essential for manned missions and is typically a tractor-type configuration with solid motors (preferred due to high reliability and high thrust levels). The Crew Module (CM) is housed inside the CES fairing at the rear portion. CES pulls CM away from the launch vehicle and takes it to a safe altitude and range in case of abort during the ascent phase. Solid motors are placed ahead where the diameter is less than the CES diameter at the base. The two regions of different diameter are joined by a flare. The flare is typically conical in shape (e.g. Soyuz, Shenzhou, Saturn, etc.). The configuration design of CES should ensure that static and dynamic load on the structure is minimum for optimizing the mass of the structure. Launch Abort System (LAS) or CES has been used in the Mercury and Apollo programmes, which were the first series of manned space missions. Both the Mercury and Apollo spacecraft used rocket-powered escape towers ${ }^{1}$. Subsequently, several changes have been made in LAS based on requirement. Figure 1 shows LAS configurations which are flown worldwide.

While designing CES, different mission abort strategies need to be finalized. Figure 2 shows a typical mission abort and its sequence. During the first stage, CES is

\footnotetext{
*For correspondence. (e-mail: jksnivas@gmail.com)
}

used in case of any emergency. This abort scenario has been further divided into three categories based on the design criteria of CES. They are: (1) launch pad abort, (2) transonic abort, and (3) high-altitude abort. During this atmospheric phase of the abort, stability of CES along with CM is essential from the safety aspect of the crew. Towards this, various configurations have been studied in detail.

This article discusses about evolution in aerodynamic design of CES and its stability aspects. Twenty-nine different configurations have been studied before finalizing a configuration. For all these configurations, detailed

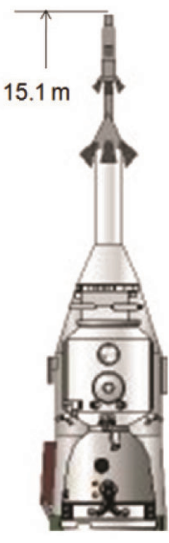

Shenzhou
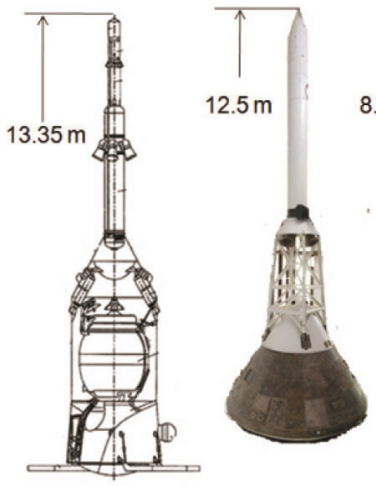

Soyuz

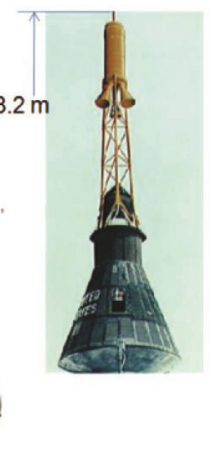

Mercury
Figure 1. Launch Abort System configuration flown worldwide.

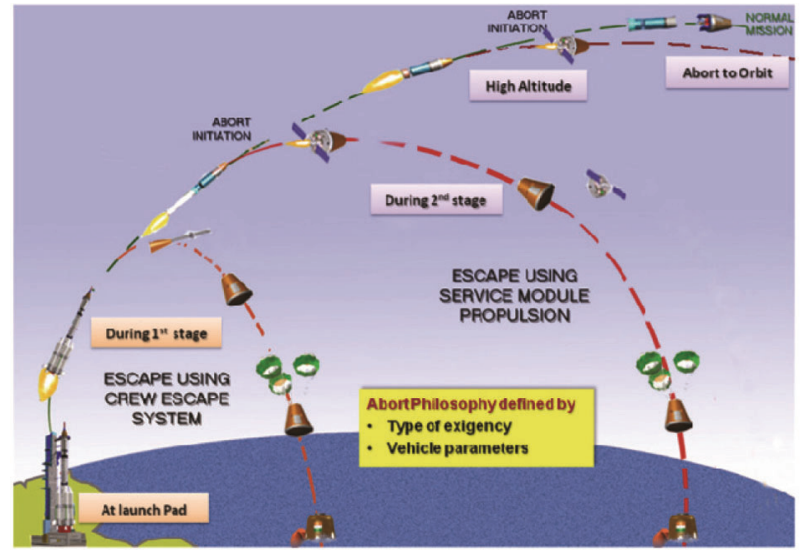

Figure 2. Mission abort and flight profile. 
computational fluid dynamics (CFD) simulations have been carried out at an angle of attack of $4^{\circ}$ under jet-off and various jet-on conditions to understand the aerodynamic behaviour at different scenarios. In-house developed code PARAS-3D has been used to carry out flow simulations $s^{2,3}$. It has the capability to predict aerodynamic coefficients of launch vehicle with conventional or reverse flow nozzle in the presence of jet ${ }^{4}$.

\section{Geometrical details of CES}

Figure 3 shows the major components of a typical CES. Description of each major component of the CES is given below.

\section{Crew Escape Motor}

The main component of CES is the Crew Escape Motor (CEM) (solid motor), which is sized and fired in such a way that $\mathrm{CM}$ is pulled away from the launch pad if there is any explosion or any undesired event at the launch pad. Similarly, if the flight is deviated away from the nominal trajectory, CEM pulls the crew from the ongoing stage at any instant of time. The nozzles of this motor are designed such that direct impingement of the jet on the CES outer structure is avoided by directing the flow away from the body. This motor gives the required acceleration during abort manoeuvre.

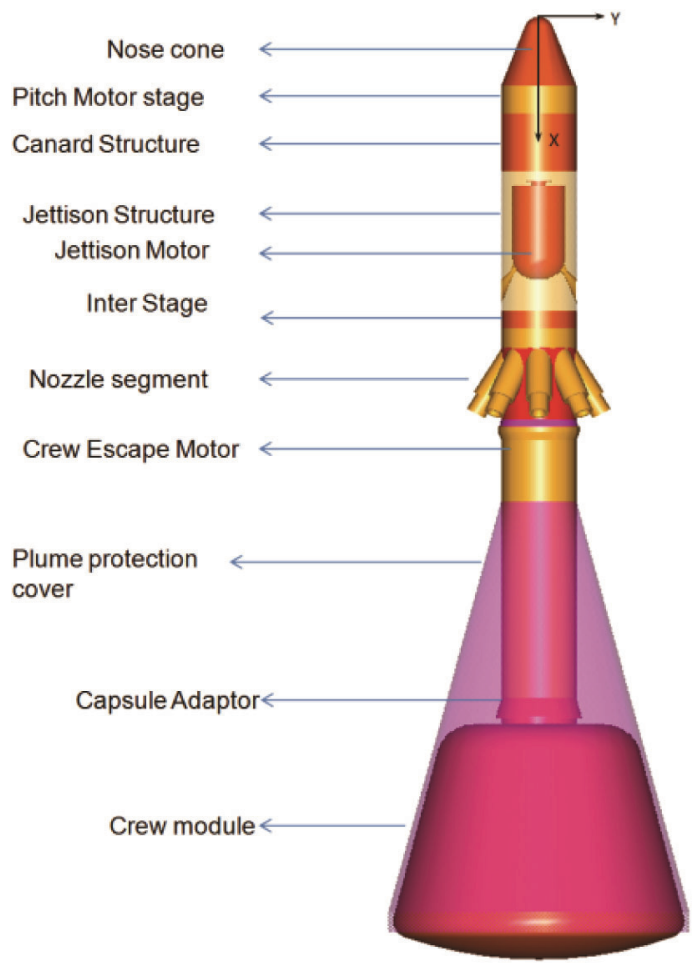

Figure 3. Different components of a typical Crew Escape System (CES).

CURRENT SCIENCE, VOL. 120, NO. 1, 10 JANUARY 2021

\section{Pitch Control Motor}

The Pitch Control Motor is fired simultaneously along with CEM to ensure positive pitching, which makes the escaping module steer away from the accelerating launch vehicle and provide sufficient range for launch pad abort.

\section{CES Jettisoning Motor}

This motor is used to separate CES and CM after proper orientation of the latter. It provides the required thrust and operates for a very short duration.

\section{Canard surfaces}

Canards are deployed to ensure that CES has a destabilizing moment to provide proper orientation of $\mathrm{CM}$ for parachute deployment.

\section{Crew Module}

$\mathrm{CM}$ accommodates the crew and their associated systems to survive in space.

\section{Aerodynamic design criteria}

Earlier it has been mentioned that the propulsive mode is used to separate CES from the ongoing vehicle. The

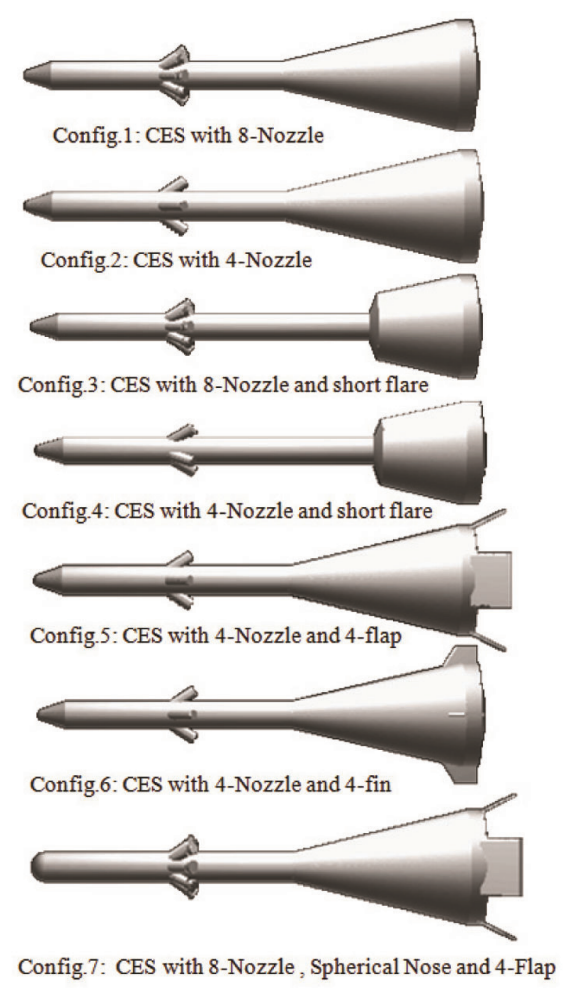

Figure 4. Configurations of CES-phase 1. 

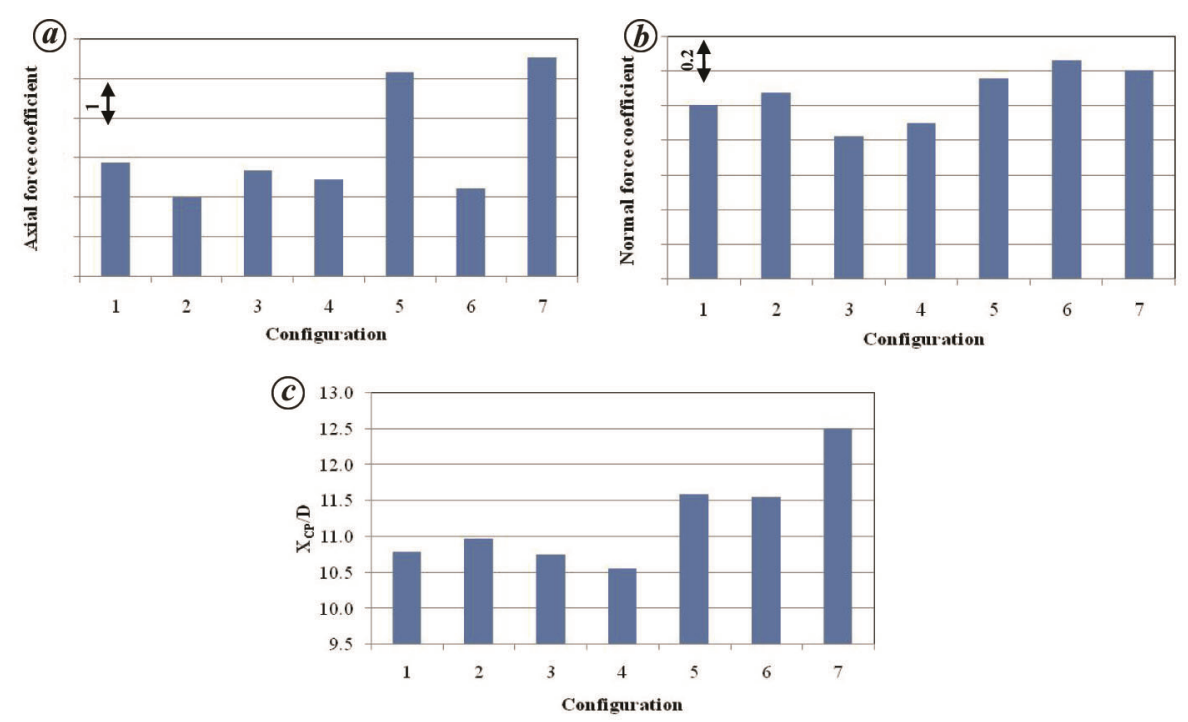

Figure 5. Aerodynamic characteristics for CES configuration-phase 1. a, Variation of axial force coefficient. $\boldsymbol{b}$, Variation of normal force coefficient. $\boldsymbol{c}$, Variation of centre of pressure.

motors are located in the middle of the vehicle. Moreover, presence of CM at the base of CES pulls the centre of gravity $\left(X_{\mathrm{cg}}\right)$ of the configuration towards CES base. The stability of the configuration is decided based on static margin (difference between centre of pressure $\left(X_{\mathrm{cp}}\right)$ and centre of gravity $\left.\left(X_{\mathrm{cg}}\right)\right)$ of the vehicle. Hence pulling $X_{\mathrm{cp}}$ behind $X_{\mathrm{cg}}$ is a big challenge from the aerodynamic aspect, keeping other aerodynamic parameters (i.e. drag, normal force, shock strength, etc.) to a minimum value. For the present study, minimum static margin requirement is considered as $1 D$, where $D$ is the motor diameter. Unlike other launch vehicle configurations, jets come out from the middle of the vehicle and aerodynamics in the presence of these jets will be significantly different. All the initial studies are carried out under jet-off condition. Having obtained a reasonable static margin from jet-off simulations, jet-on simulations are done to finalize the size of the configuration.

\section{Phase-1 studies}

Based on the literature survey and CES used by different agencies, various configurations of CES are arrived at that encapsulate $\mathrm{CM}$ and other required propulsion units. Detailed studies have been carried out by changing the number of nozzles, shaping of plume protection cover (PPC), adding fins and flaps at the base to meet the stability requirement, etc. Seven different configurations have been analysed in the first stage (Figure 4). The cone angle is chosen such that the nozzle angle and cone are similar to avoid direct plume impingement on CM. Detailed flow simulations (using CFD) have been carried out for all these seven configurations at typical Mach number of 0.60 at an angle of attack of $4^{\circ}$ under jet-off condition.
Figure $5 a$ and $b$ shows variation of axial force and normal coefficient of these configurations. It is observed that configuration 5 and 7 have higher drag due to the presence of flaps at the base of the vehicle, whereas the rest are about $50 \%$ less drag compared to configurations 5 and 7. As the flare length increases normal force coefficient increases. Configurations 3 and 4 have the least normal force compared to other configurations. Addition of fins and flaps further increases the normal force coefficient for configurations 5-7 compared to configurations 1 and 2.

Figure $5 c$ shows variation of centre of pressure. It is observed that configurations with fins or flaps have $X_{\mathrm{cp}}$ closer to the base compared to other configurations. Hence, they will have more static aerodynamic stability, as expected. This indicates that in order have $X_{\mathrm{cp}}$ towards the base, fins or flaps are mandatory. Based on $X_{\text {cg }}$ location of each configuration, none of the configurations is found to meet the minimum static margin requirement.

Analysis of data for configurations with four and eight nozzles shows that the drag is more for the latter configuration due to more surface area of 8 protruding nozzles. Moreover, it also results in $X_{\mathrm{cp}}$ towards the nose due to additional lifting surface provided by the nozzle. Hence it is more unstable compared to four-nozzle configuration. Hence eight-nozzle configuration is not considered here.

The requirement of fins or flaps can be avoided if an active control surface is present. With the help of control surface, CES will take CM to the required attitude before separation. Adding control or canard surface is one of the solutions towards this. The rocket must be stable when the canards are not deployed, and must become unstable when the canards are deployed so as to manoeuvre the vehicle to separate CM from CES at the desired attitude easily. The analysis has been carried out for configuration 
under the deployment of canard surface (Figure 6) to make the vehicle unstable, so as to manoeuvre it. The canard system must be designed for deployment under high dynamic pressure, and must also withstand heating associated with the flow. The flow simulations were carried out over this rocket configuration with and without canard to analyse the flow.

Figure 7 shows the variation of $X_{\mathrm{cp}}$ with and without deployed canard. It is observed that, due to canard deployment, vehicle $X_{\mathrm{cp}}$ moves towards the nose and vehicle becomes unstable ${ }^{5}$. The point to be noted here is, canards can be effectively used to reorient the vehicle so as to have an easy separation of CM. But later based on system

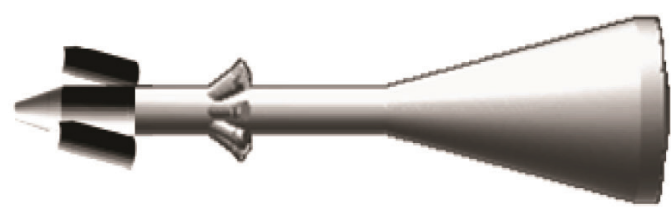

Figure 6. Configuration 1 with deployed canard.

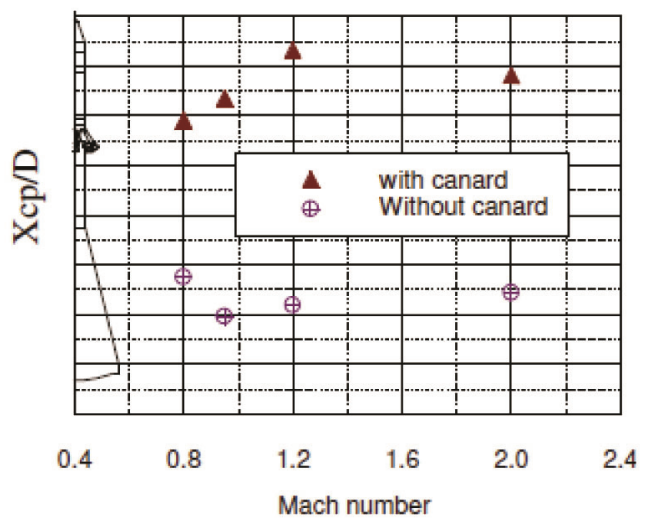

Figure 7. Variation of $X_{\mathrm{cp}}$ without and with canard configuration.

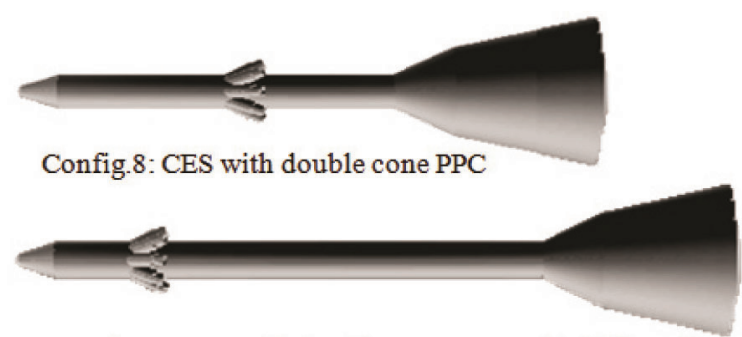

Config.9: CES with double cone PPC and additional interstage

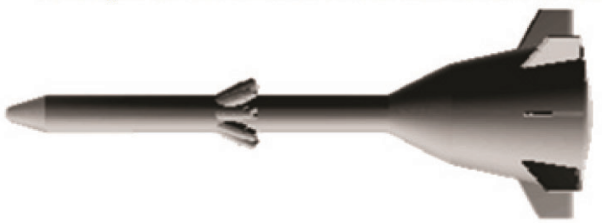

Config. 10: Configuration 8 with 8 -Fin

Figure 8. Configurations of CES-phase 2. requirement, this canard configuration has been discarded.

From the above mentioned studies, it is observed that, change in the static margin for four or eight nozzles configuration is minimal. Addition of fins and flaps moves $X_{\mathrm{cp}}$ towards the base. Studies also show that canards are effective to reorient the vehicle for easy separation of CM.

\section{Phase-2 studies}

As conical-shaped PPC produces higher drag, bi-conic configuration is chosen which produces comparatively lower drag than a single cone within the same envelope. Figure 8 shows the configurations studied in this phase. Configuration 8 is CES with double cone PPC and in configuration 9 CES motor is located near the nose to have less impact of jet on PPC and one inter-stage added to see its effectiveness. Configuration 10 is arrived at by adding eight fins to configuration 8 to improve stability.

From the flow simulations over these configurations it is observed that though there is a reduction in drag due to bi-cone, $X_{\mathrm{cp}}$ moves forward due to addition of secondary cone. Extending the motor length in configuration 9 does not improve the static margin significantly. For both the configurations there is no lifting surface available at the base of the vehicle. So, they are unable to meet the required static margin criteria and hence discarded from further studies.

Configuration 10 has fins at the base of the vehicle. Simulations carried out at various Mach numbers under jet-off and jet-on conditions show that the vehicle meets the stability under jet-off condition (Figure 9). However, the configuration is unstable above Mach number 2.2 jeton condition. To understand the reason for this, flow field at higher Mach number is analysed.

Figure 10 shows Mach palette under jet-off and in the presence of jet for configuration 10 at higher Mach number $(M=4.0)$. Under jet-off condition at higher Mach

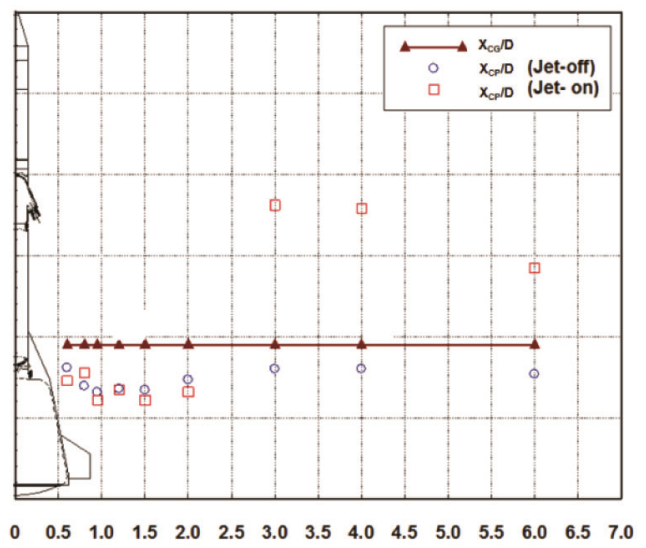

Figure 9. Variation of centre of pressure under jet-off and jet-on condition for configuration 10 . 
number shock with high strength is formed at the nose cone, ahead of the nozzle flare region and ahead of the PPC cover. The wake region is observed at the base of the vehicle at all Mach numbers. At high supersonic Mach numbers, the jet pressure ratio at nozzle exit being very high (pressure ratio at $M=4$ is 945 ), leads to completely engulfing the base portion of the vehicle ${ }^{6,7}$. Due to huge expansion of the jet, downstream portion of the configuration (double cone and fins) loses its lift and the centre of pressure moves towards the nose making the vehicle more unstable compared to jet-off case. Also, the main disadvantage of this configuration is the movement of centre of pressure of complete ascent vehicle towards the nose by $2.5 \mathrm{D}$ due to the presence of fixed planner fins. Hence grid fin $^{6}$ and tower configuration options are considered (Figure 11). The advantage of grid fin is that it can be folded during ascent flight and will be deployed only under abort conditions or when required.

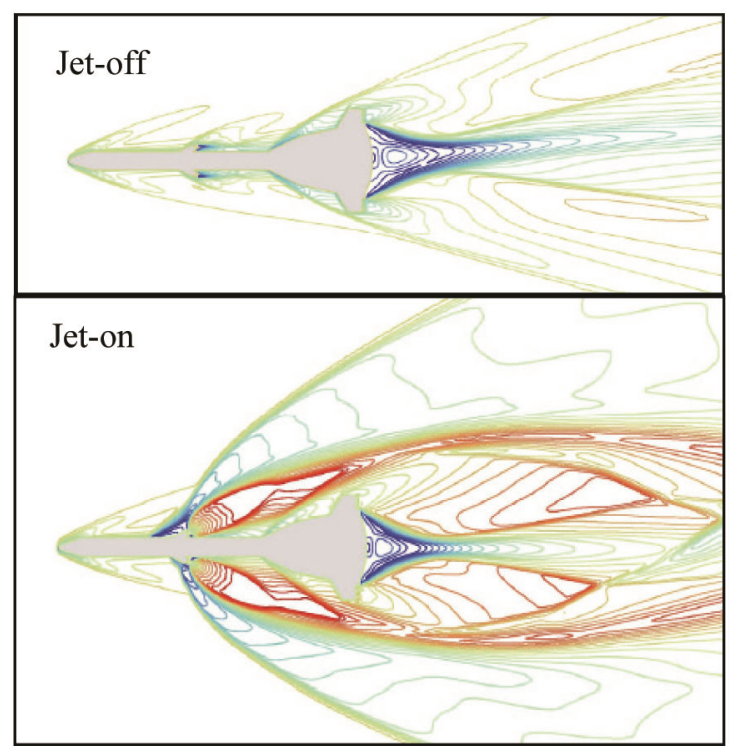

Figure 10. Mach isoline without and with jet for config. 10 at Mach number 4.0 and $\alpha=4^{\circ}$.
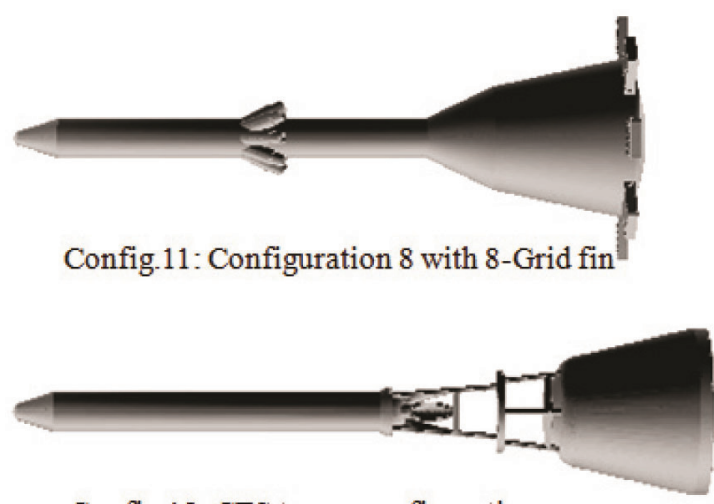

Config.12: CES tower configuration

Figure 11. Configuration of CES-phase 2(a).
Flow simulations over these configurations are carried out at various Mach numbers under both jet-on and jet-off conditions. Analysis of the flow field and aerodynamic coefficients shows that under jet-off condition, CES configuration with grid fin (config. 11) and CES configuration with tower (config. 12) meet the requirement of static margin (Figures 12 and 13 respectively). However, assessment under jet-on condition shows that above Mach number 2.2 both the configurations are unstable. In order to overcome this situation, an attempt was made to have motors mounted at the base for higher supersonic Mach numbers (high altitudes), or all the altitudes (all Mach numbers) so that the effectiveness of the body and fin does not reduce significantly in the presence of jets.

Based on the above studies, it is clear that the presence of fin is essential and the planner fin does not satisfy the requirement of static margin. Hence grid $\operatorname{fin}^{8}$ is selected as lift-producing device for further studies. At the same

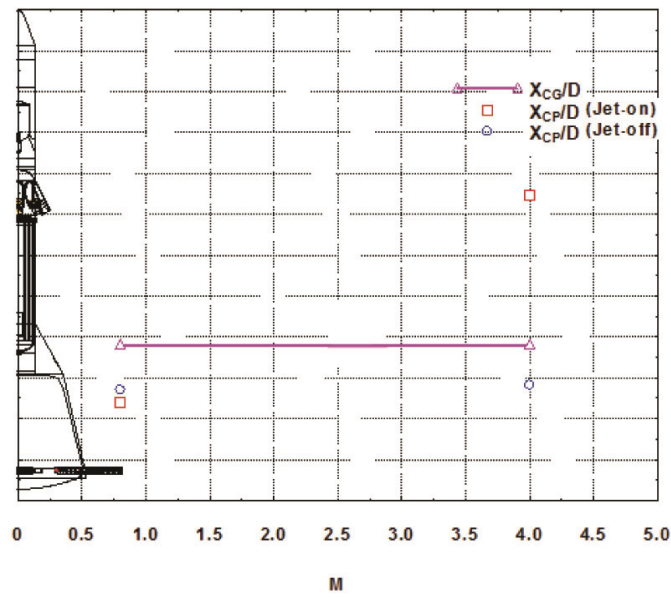

Figure 12. Variation of centre of pressure under jet-off and jet-on condition for configuration 11 .

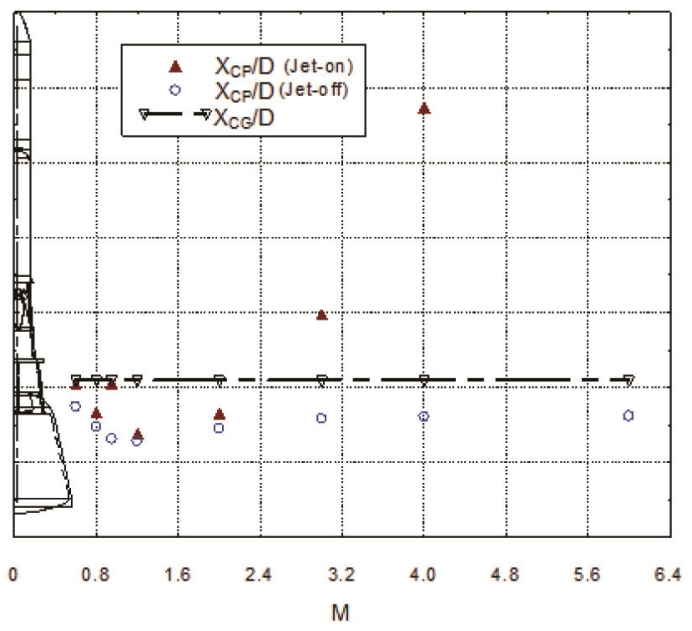

Figure 13. Variation of centre of pressure under jet-off and jet-on condition for configuration 12 .

CURRENT SCIENCE, VOL. 120, NO. 1, 10 JANUARY 2021 
time, tower-type concept also works fine. But at higher Mach number, to meet the margin, motors are to be mounted at the base region of the vehicle.

\section{Phase-3 studies}

Based on the above understanding, motors are mounted at the base of the configurations along with grid fins to arrive at four different configurations (Figure 14). Simulations were carried out under jet-off and jet-on conditions at various Mach numbers. It is noticed that, configurations 14-16 are unstable under jet-off condition. As the heavy $\mathrm{CM}$ sits at the bottom of the vehicle, $X_{\mathrm{cg}}$ lies very close to the vehicle base (at a distance of $\sim 25 \%$ of the vehicle length). Even though grid fins are present, they are not sufficient to shift $X_{\mathrm{CP}}$ towards the base. A bigger grid fin may work, but it will be difficult to fold along the surface during ascent. Hence, these confirmations were discarded from further analysis. Configuration 13 is statically stable under jet-off and jet-on conditions. However, in order to meet the requirement of minimum static margin, grid fin size needs to be increased accordingly.

Different tower-type configurations are also studied to move $X_{\mathrm{cg}}$ towards the nose. Configurations 17-19 are tower configurations with high-altitude motors kept on the CM. Grid fins are introduced in configuration 20 to

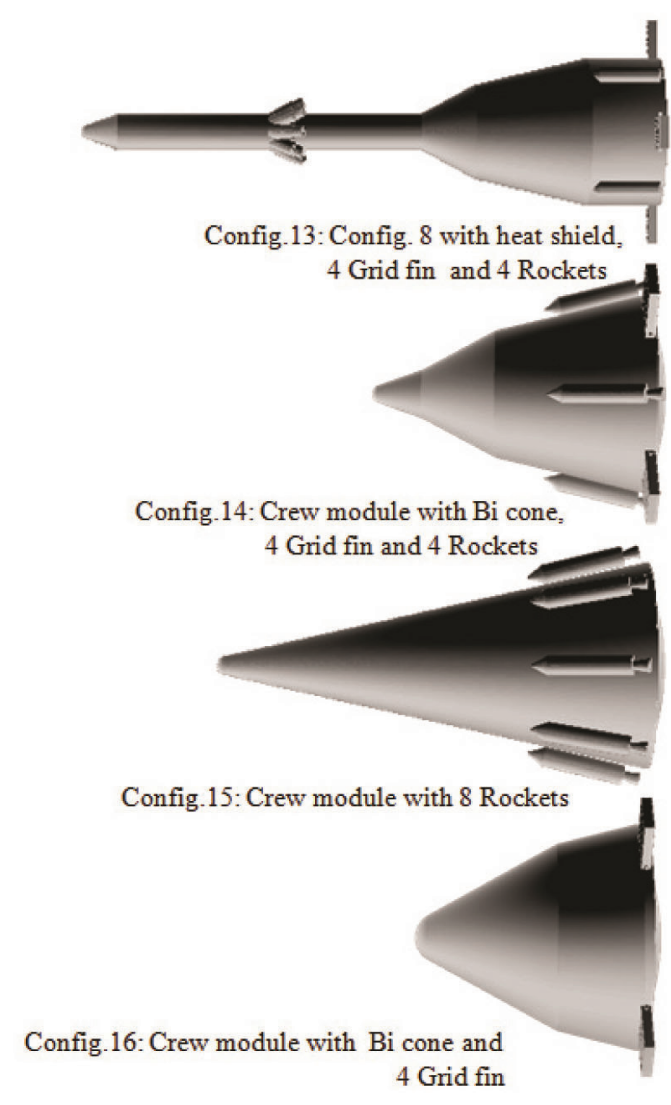

Figure 14. Configuration of CES-phase 3(a). improve the stability of the vehicle (Figure 15). Simulations are carried out at various Mach numbers ranging from 0.8 to 4 at an angle of attack of $4^{\circ}$. All the configurations are found to be statically stable under jet-off condition. In the presence of forward jet (CEM1), configuration 18 is stable up to Mach 2.5 but does not meet the minimum static margin requirement of $1 D$. Configuration 19 meets the static stability requirement in the presence of CEM1 up to Mach 2.5, while above Mach 2.5, high-altitude motors (CEM2, i.e. motors at the base region) are used and the vehicle is found statically stable. But aero-acoustic environment on $\mathrm{CM}$ will be higher during the CEM1 jet-on phase. Adding PPC may help for this (configuration 21).

Configuration 20 consists of CM with increasing CM Fairing diameter and four grid fins with an aim that the lift generated by the base region will be higher leading to backward shift of $X_{\mathrm{cp}}$ and the vehicle will have sufficient static margin. Solid motors are housed inside and $45^{\circ}$ away from the grid fins to improve its performance. Configuration 21 is a combination of configurations 20 and 12. It consists of two sets of motor one for low altitude and the other for high altitude. Figure 16 shows Mach palette for configuration 20 in the presence of the jet. As the jet exit is located in the cylindrical region, full expansion occurs at the downstream of the geometry, which does not affect the aerodynamics like earlier cases (where the motors are located on the top). Simulation results show that configurations 20 and 21 are statically stable under jet-off and jet-on conditions, but does not meet the

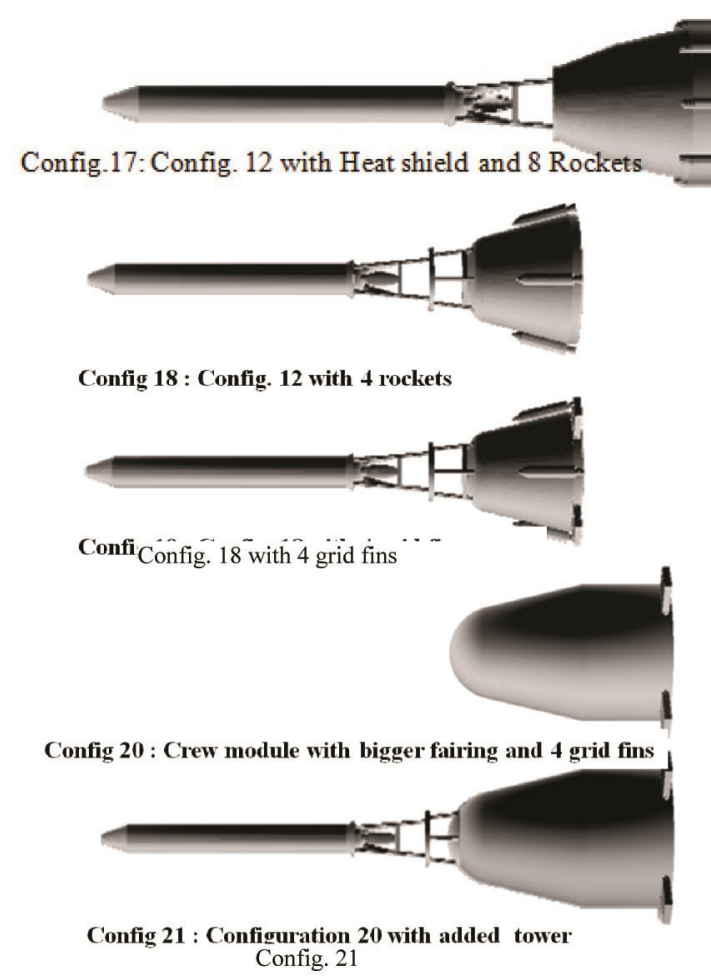

Figure 15. Configuration of CES-phase 3(b). 
static margin requirement of $1 D$ in the presence of jet. Improving the grid fin size can increase the static margin of the vehicle. But these types of configurations need large pull-out length $(3 \mathrm{~m})$ to separate CM from CES.

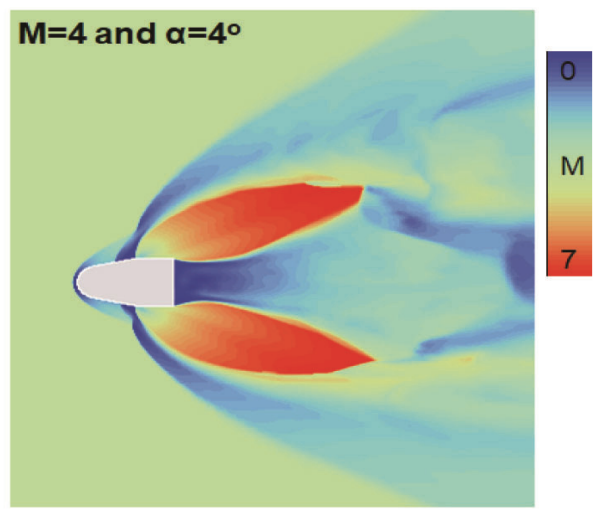

Figure 16. Mach palette at Mach number 4.0 for configurations 21 .

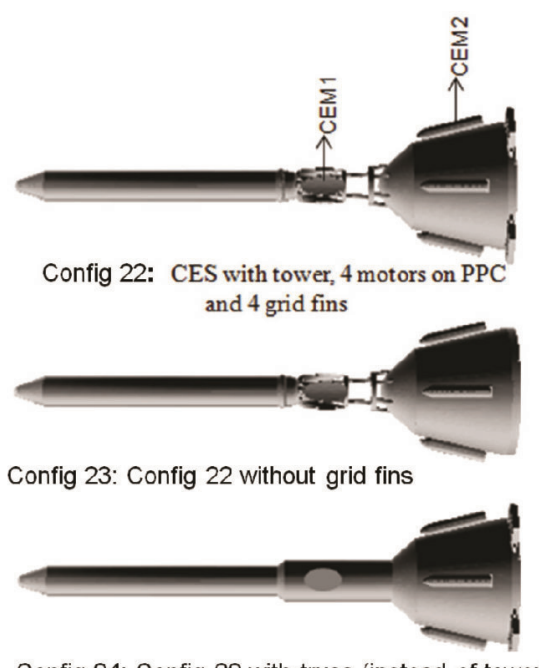

Config 24: Config 22 with truss (instead of tower)

Figure 17. Configuration of CES with high-altitude motors.

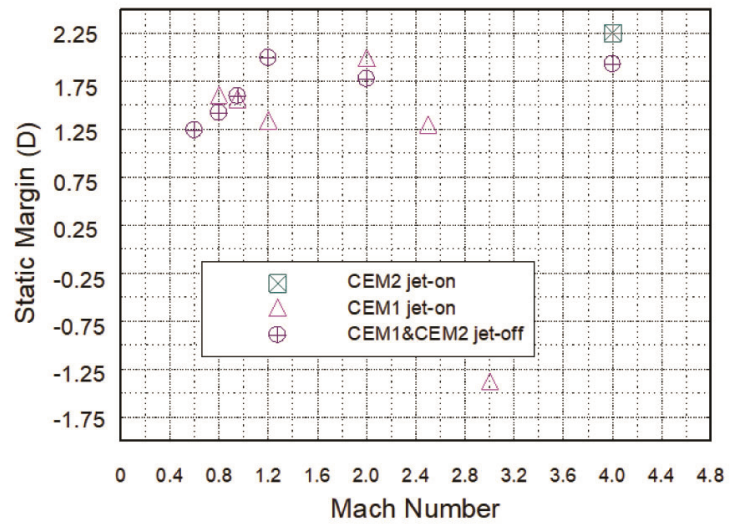

Figure 18. Variation of static margin with Mach number for jet-off and various jet-on conditions for configuration 22 .
After studying various options, particularly to meet aerodynamic stability requirement, configuration 22 has been arrived at which has independent motors for low and high altitude, tower configuration and grid fins (Figure 17). Aerodynamic analysis has been carried out at various Mach numbers under jet-off, CEM1 jet-on and CEM2 jet-on conditions. It is observed that vehicle is statically stable under jet-off condition (Figure 18). Up to Mach number 2.5, CES is statically stable under CEM1 jet on condition and above Mach 2.5 the vehicle is stable in the presence of CEM2 jet-on condition, which shows the requirement of high-altitude motor (CEM2). This configuration meets all the stability requirement criteria. For

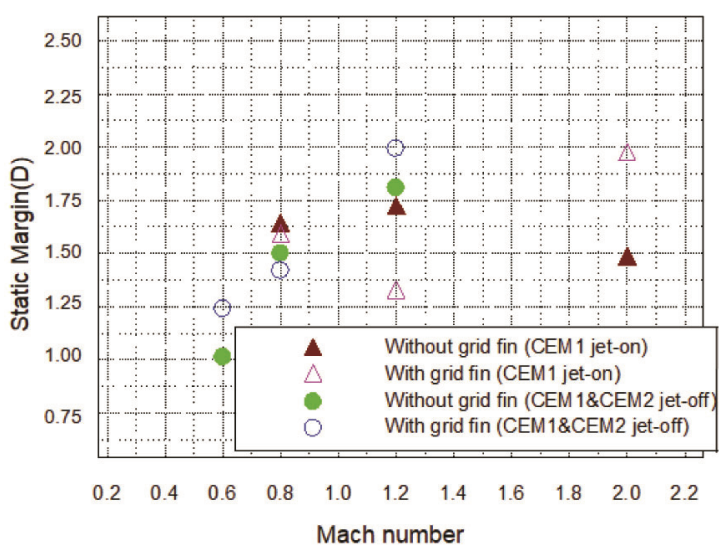

Figure 19. Effect of grid fins on static margin - configurations 22 and 23 .
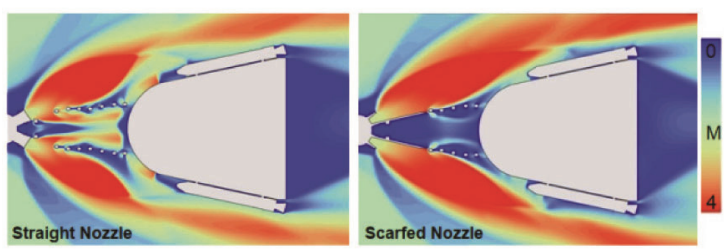

Figure 20. Mach palette for configuration 22 with straight and scarfed nozzle.

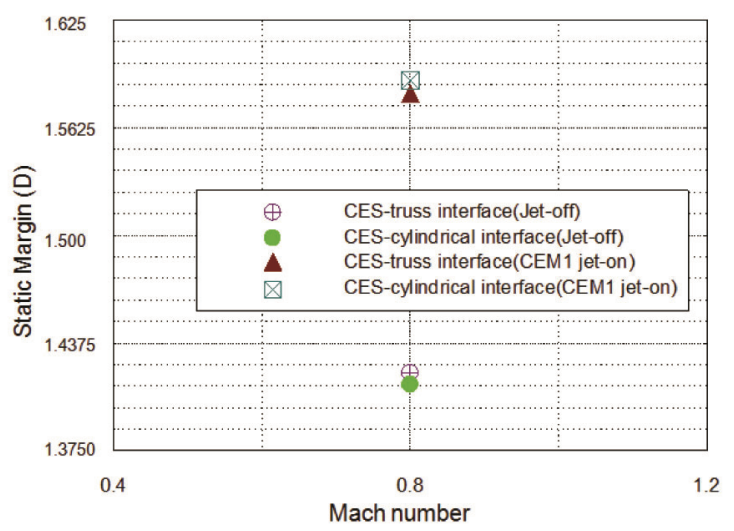

Figure 21. Variation of static margin for configurations 22 and. 24 .

CURRENT SCIENCE, VOL. 120, NO. 1, 10 JANUARY 2021 
this configuration, studies are carried out with and without grid fins (configuration 23) to estimate the effectiveness of grid fins. It is observed that the grid fin improves stability by $0.675 D$ at higher Mach number (Figure 19). However, the presence of grid fin increases the mass and moves $X_{\text {cg }}$ forward by $0.175 D$. Hence, the net benefit of adding grid fin is $\sim 0.5 D$ in static margin at $M>2.50$.

Studies are also carried out by firing both the motors simultaneously at low altitudes. Firing of two motors together helps in two ways. First, the centre of gravity $(\mathrm{CG})$ moves forward because CEM2 motors located behind CG burning of these motors moves $X_{\mathrm{cg}}$ towards nose. Second, the centre of pressure moves backward due to turning of flow within. It is observed that at Mach number 0.8 , to both jet firing together improved the static margin by $0.25 D$. Based on this study, it has been decided

Table 1. Important features of finalized Crew Escape System configuration

\begin{tabular}{ll}
\hline Component & Features/dimensions \\
\hline Nose cone angle & $20^{\circ}$ \\
Pitch Motor & 1 no. \\
LEM diameter & $1 D$ \\
LEM nozzles & 4 nos \\
HEM nozzles & 4 nos \\
CJM nozzles & 4 nos \\
CM shroud shape & Conical \\
CM Shroud angle & $30^{\circ}$ \\
CM Fairing & $4.625 D$ \\
Grid fins & 4 nos \\
Grid fin dimensions & $1.875 D^{*} 1.875 D$ \\
Overall length & $18 D$ \\
\hline
\end{tabular}

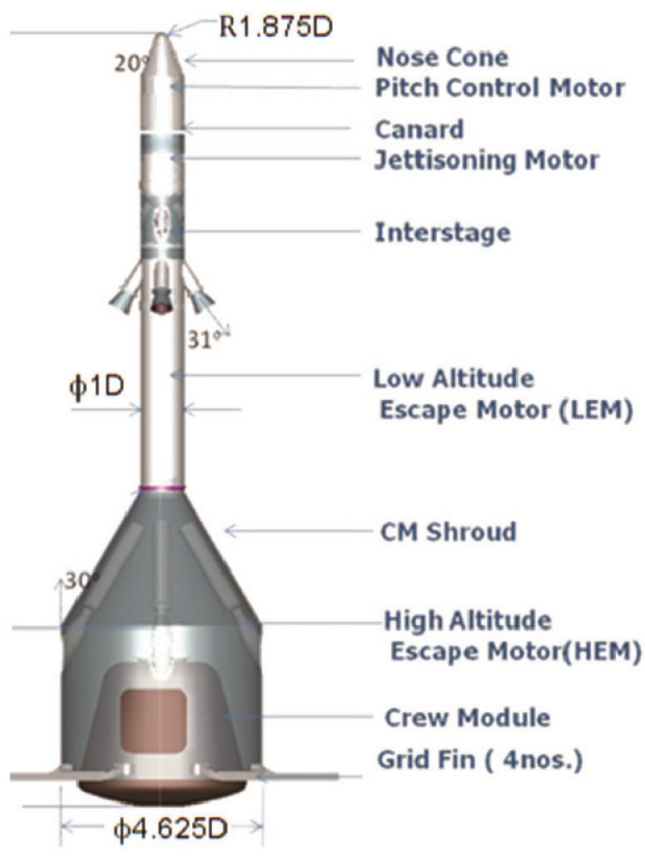

Figure 22. Finalized configuration for CES. that for low-altitude two sets of motors will be fired simultaneously, but at high altitude only CEM2 will be fired.

Effect of straight and scarfed nozzle on static margin is studied for configuration 22 at Mach number 2.5 under CEM1 jet-on condition. Figure 20 shows Mach palette for these two nozzle configurations. It is observed that for the straight nozzle, part of the jet impinges on top of the PPC and downstream of the jet also closer to the body, which leads to loads on the body compared to scarfed nozzle geometry where jet impingement is not observed. Scarfed nozzle geometry has centre of pressure of $0.66 \mathrm{D}$ higher than straight nozzle geometry at Mach 2.5. Hence it is selected.

Configuration 24 shows cylindrical configuration instead of tower. The merits of cylindrical against tower configuration are assessed at Mach number 0.8 . Figure 21 shows the results of static margin under jet-off and CEM1 jet-on. It is observed that both configurations have similar stability margin. So, cylindrical configuration is chosen from simplicity aspect of the design.

Once the requirement of separate low-altitude escape motor (LEM) and high-altitude escape motor (HEM) has been established, detailed studies are carried out to finalize the locations of these motors. If the low-altitude motors are close to $\mathrm{CM}$, it increases the vibro acoustic and thermal loads on the latter. HEMs should be placed such that the jet does not affect grid fin performance significantly due to pluming, specially at higher altitude.

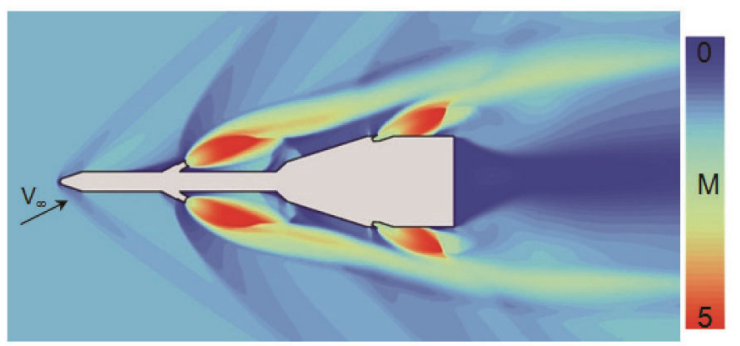

Figure 23. Typical Mach palette at Mach 2.5.

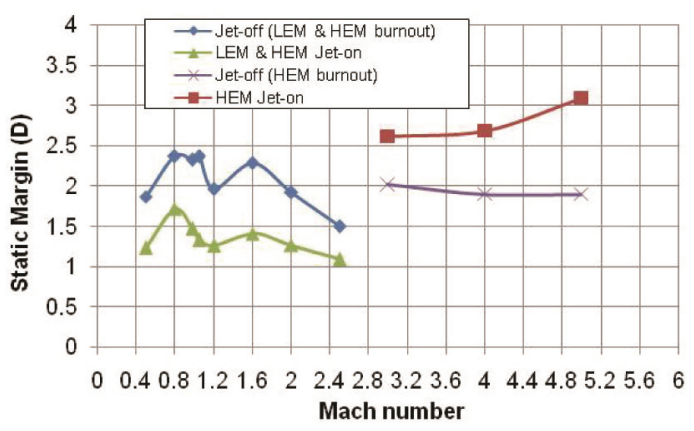

Figure 24. Static margin variation under various jet-on conditions. 
After finalizing the shape, studies are carried out to improve the performance of the grid fin by varying different geometric parameters of the grid fin (i.e. chord length, span, cell spacing, web shape, etc.). After detailed studies, the configuration with double wedge with $1.875 D$ width, $1.875 D$ span and $0.1875 D$ chord grid fin has been chosen (Figure 22).

Based on the above studies, CES configuration has been finalized. Table 1 shows the major features of the selected configuration. For the finalized configuration (configuration 23), CFD simulations are carried out under jet-off, LEM and HEM under jet-on and HEM alone under jet-on condition for Mach numbers 0.4-6.0 at an angle of attack of $4^{\circ}$. Figure 23 shows the typical Mach palette for the finalized configuration at Mach 2.5 under LEM and HEM jet-on. All the flow features like shock, jet boundary and wake flow are captured well. The vehicle is statically stable under jet-off condition for all Mach numbers. Up to Mach number 2.5, CES is statically stable under LEM and HEM jet-on condition and above Mach 2.5 the vehicle is stable in the presence of HEM jet-on condition. Figure 24 shows variation of the static margin plot. For various conditions minimum static margin of $1 D$ achieved.

\section{Conclusion}

Various geometrical parameters have been studied in order to finalize the CES configuration. The $30^{\circ}$ conical $\mathrm{CM}$ shroud has been selected after studying various options (conical, bi-cone and heat shield type). Grid fins are selected over conventional fins. Grid fins will be deployed only under abort conditions. Reverse-flow nozzles are chosen in order to avoid direct jet impingement on the $\mathrm{CM}$ shroud. In order to ensure stability of the vehicle under all flight regimes two sets of motors, namely LEM and HEM are introduced and placed at appropriate loca- tions. Based on CFD studies, the chosen configuration is found to be statically stable under jet-off and LEM and HEM jet-on conditions up to Mach number 2.5 and stable above Mach 2.5 in the presence of HEM jets.

1. McCarthy, J. F., Ian Dodds, J. and Crowder, R. S., Development of the Apollo Launch Escape System. J. Space Craft, 1968, 5(8), 927932.

2. Ashok, V. and Babu, T. C., Parallisation of Navier-Stoke code on a cluster of workstations. In Proceedings of the 6th International High Performance Computing Conference. Lecture Series in Computer Science, Springer Verlag, 1999.

3. Balu, R., Babu, T. C., Ashok, V., Pradeep Kumar and Das, D., Development of low cost parallel computing platform for CFD applications. In Third AeSI Annual CFD Symposium, 11-12 August 2002.

4. Sreenivasulu, J. et al., CFD analysis of a Launch vehicle with reverse flow nozzles - a validation study. Int. J. Aerospace Innov., 2013, 5(1).

5. Sreenivasulu, J. et al., CFD analysis over a rocket configuration with and without canard. In 12th Annual CED Symposium, Bangalore, 11-12 August 2010.

6. Aftosmis, M. J. and Rogers, S. E., Effects of jet-interaction on pitch control of a launch abort vehicle. AIAA Paper 2008-1281.

7. Sreenivasulu, J. et al., Flow field analysis over a typical rocket with and without jet. In 10th Asian Symposium on Visualization, SRM University, Chennai, India, 1-5 March 2010.

8. Sreenivasulu, J. et al., Aerodynamic analysis of a rocket configuration with grid fin. In Proceedings of the 37th National and 4th International Conference on Fluid Mechanics and Fluid Power, IIT Madras, Chennai, India, 16-18 December 2010.

ACKNOWLEDGEMENTS. We thank the reviewers of the configuration during 2009-2018. S. V. Sharma (former DD, AERO), Dr K. Sivan (former DD, AERO), S. Swaminathan (former DD, AERO), and S. Pandian (former DD, AERO). Madanlal (Chairman, SCR), S. Ramakrishnan (former AD Projects, VSSC; Chairman, LVDRT; Chairman, PEX mission and CES). We also thank C. K. Rakesh (Sci./Engr 'SF', VSSC) for providing various $3 \mathrm{D}$ model geometries.

doi: $10.18520 / \mathrm{cs} / \mathrm{v} 120 / \mathrm{i} 1 / 96-104$ 